\title{
Experiência Inicial de um Novo Centro no Brasil na Abordagem Híbrida para a Síndrome de Hipoplasia do Coração Esquerdo
}

\author{
Maurício Jaramillo Hincapie, Leonardo Cogo Beck¹, Cristina Camargo Afiune , Juliana Duarte Diniz, \\ Fernando Antibas Atik¹, Jorge Yussef Afiune' ${ }^{1}$, Luiz Fernando Canêo ${ }^{1}$
}

\section{RESUMO}

Objetivo: Apresentar os resultados iniciais com o emprego do procedimento híbrido na síndrome de hipoplasia do coração esquerdo (SHCE). Método: Oito pacientes com SHCE foram submetidos ao primeiro estágio do procedimento. As indicações foram: preferência do grupo em quatro $(50 \%)$ pacientes, infecção sistêmica em evolução em dois $(25 \%)$, disfunção ventricular grave em um $(12,5 \%)$ e lesão encefálica grave com hipertensão pulmonar em um (12,5\%). Resultados: Três $(37,5 \%)$ pacientes morreram no primeiro estágio, dois (25\%) foram submetidos ao segundo estágio, e nenhum foi submetido ao terceiro estágio. Três $(37,5 \%)$ crianças aguardam o segundo estágio, e duas delas estão internadas, uma por insuficiência respiratória grave e infecção sistêmica e outra pós-atriosseptostomia com colocação de stent, evoluindo com infecção pulmonar. Reintervenções foram necessárias em 50\% dos casos, em dois deles mais de uma vez (atriosseptostomias por balão em três e colocação de stent na comunicação interatrial em um). O tempo médio de ventilação no primeiro estágio foi de 585 horas e de internação na unidade de terapia intensiva, de 32 dias. Duas crianças já foram submetidas ao segundo estágio e ambas morreram: morte súbita em uma e durante dilatação da artéria pulmonar esquerda por estenose residual em outra. Conclusão: A curva de aprendizado, a dificuldade técnica da realização do segundo estágio, a necessidade de múltiplas intervenções interestágios e as sequelas anatômicas deixadas pelos procedimentos prévios reforçam que o procedimento híbrido deve ser considerado tão complexo quanto a operação de Norwood.

DESCRITORES: Síndrome do coração esquerdo hipoplásico/ cirurgia. Angioplastia. Cardiopatias congênitas. Stents. Artéria pulmonar/cirurgia.

\author{
ABSTRACT \\ Hybrid Approach for Treatment of the Hypoplastic \\ Left Heart Syndrome: Initial Experience \\ of a New Center in Brazil
}

Objective: Our objective was to review the early results of hybrid procedures in hypoplastic left heart syndrome (HLHS) at our institution. Methods: Eight HLHS patients were submitted to the first stage of the procedure and the indications were the surgeon's preference in $4(50 \%)$, sepsis in $2(25 \%)$, severe ventricular dysfunction in $1(12.5 \%)$ and severe brain damage with pulmonary hypertension in the latter $(12.5 \%)$. Results: Three $(37.5 \%)$ patients died after the intervention, $2(25 \%)$ underwent the second stage and none underwent the third stage. Three $(37.5 \%)$ patients are currently waiting for the second stage and 2 of these are currently hospitalized: one with pneumonia-related sepsis and the other with pneumonia post atrial septal defect stenting. Reinterventions were necessary in $50 \%$ of the cases, more than once in 2 patients (balloon atrioseptostomy in 3 and atrial septal defect stenting in 1). Mean mechanical ventilation duration in the first stage was 585 hours and mean hospitalization time in the intensive care unit was 32 days. Both patients undergoing the second stage died: sudden death in one and residual left pulmonary artery stenosis after percutaneous dilatation in the other. Conclusion: Hybrid procedures should be considered as complex as Norwood operation due to the learning curve, technically difficulties to perform the second stage, need of multiple interstage reinterventions and anatomic sequelae related to previous procedures.

DESCRIPTORS: Hypoplastic left heart syndrome/surgery. Angioplasty. Heart defects, congenital. Stents. Pulmonary artery/surgery.

\footnotetext{
1 Instituto de Cardiologia do Distrito Federal - Fundação Universitária de Cardiologia - Brasília, DF, Brasil.

Correspondência: Maurício Jaramillo Hincapie. SQSW 302 - bloco D - ap. 422 - Sudoeste - Brasília, DF, Brasil - CEP 70673-204 E-mail: maurojara70@hotmail.com

Recebido em: 21/6/2009 • Aceito em: 26/8/2009
} 
0 tratamento do ventrículo único funcional com fluxo sistêmico dependente do canal, em especial a síndrome de hipoplasia do coração esquerdo, continua sendo um enorme desafio.

O transplante cardíaco é uma alternativa viável, porém é influenciado por preferências institucionais e, principalmente, pela disponibilidade de doadores ${ }^{1}$. Progressos significativos foram alcançados na paliação cirúrgica, no princípio de Norwood e variações, em especial no entendimento de aspectos fisiológicos da circulação em paralelo ${ }^{2}$, base de modificações na técnica cirúrgica, na condução da anestesia, da circulação extracorpórea e do cuidado pós-operatório. Atualmente, a operação de Norwood modificada é o procedimento de escolha no tratamento do neonato com síndrome de hipoplasia do coração esquerdo ${ }^{3}$.

Entretanto, os bons resultados com a cirurgia de Norwood são restritos a poucos centros com grande volume de pacientes, mesmo no primeiro mundo. Enquanto nos principais centros do mundo a mortalidade do primeiro estágio e cumulativa dos três estágios é, respectivamente, de $16 \%$ a $35,5 \%$ e de $34 \%$ a $40 \%{ }^{4-6}$, as melhores séries brasileiras publicadas demonstram $36,4 \%$ de mortalidade no primeiro está$\mathrm{gio}^{7}$, e grande parte dos centros apresenta mortalidade superior a $60 \%$, segundo dados do Departamento de Informática do Sistema Único de Saúde (DATASUS) ${ }^{8}$.

O implante de stent no canal arterial aliado a bandagem seletiva das artérias pulmonares e, se necessário, atriosseptostomia foi idealizado por Gibbs et al. ${ }^{9}$, em 1993, como outra forma de paliação da síndrome de hipoplasia do coração esquerdo. Também conhecido como procedimento híbrido decorrente da cooperação entre cirurgia cardíaca e cardiologia intervencionista, tem ganho especial interesse nos últimos anos em vários centros mundiais. Esse interesse justifica-se pela não-utilização de circulação extracorpórea no período neonatal, com diminuição das complicações pós-operatórias decorrentes desse procedimento $^{10}$, e por ser tecnicamente mais simples e contribuir para a obtenção de melhores resultados, em especial naqueles centros com resultados insatisfatórios com a operação de Norwood ${ }^{11}$.

Nosso programa de cirurgia cardíaca pediátrica iniciou suas atividades em 2005, e por se tratar de um serviço de referência em nossa região recebemos uma série de casos consecutivos com diagnóstico de síndrome de hipoplasia do coração esquerdo assim que começamos a funcionar. Isso nos trouxe grande dificuldade técnico-operacional e um resultado bastante insatisfatório com essa doença. A publicação da experiência internacional com o procedimento híbrido, seus bons resultados iniciais e a facilidade técnica apresentada estimularam as equipes clínica, cirúrgica e intervencionista pediátrica a acreditar que esse procedimento deveria ser nossa primeira escolha no tratamento da síndrome de hipoplasia do coração esquerdo.
O objetivo deste trabalho é relatar nossa experiência inicial com o emprego do procedimento híbrido num centro terciário nacional, com ênfase nos detalhes técnicos e no manuseio pré-operatório, discutindo suas vantagens e desvantagens.

\section{MÉTODO}

Estudo retrospectivo, realizado em um serviço terciário de cirurgia cardíaca do Distrito Federal, por meio da análise de prontuários de crianças submetidas ao procedimento híbrido, no período de abril de 2005 a abril de 2008. O estudo foi aprovado pelo Comitê de Ética e Pesquisa do Instituto do Coração do Distrito Federal e segue os princípios da declaração de Helsinque.

Nesse período, foram admitidos 17 pacientes portadores de cardiopatias congênitas com fisiologia univentricular e fluxo sistêmico dependente do canal. Todos os pacientes eram portadores de síndrome de hipoplasia do coração esquerdo, exceto um, cujo diagnóstico era de ventrículo único funcional com dupla via de entrada de ventrículo esquerdo, discordância ventriculoarterial, coartação de aorta e comunicação interventricular restritiva.

A operação de Norwood modificada foi realizada em 9 (53\%) pacientes e o procedimento híbrido em 8 $(47 \%)$, constituindo a população deste estudo. As indicações do procedimento híbrido foram relacionadas a preferência do grupo em 4 (50\%) pacientes, septicemia em 2 (25\%), disfunção ventricular importante em 1 e lesão encefálica grave com hipertensão pulmonar no último.

A média de idade foi de $19 \pm 12$ dias ( 4 a 62 dias), o peso médio foi de 3,4 $\pm 0,7 \mathrm{~kg}(2,3 \mathrm{~kg}$ a 4,6 kg) e não houve predomínio em relação ao sexo. Todas as crianças nasceram a termo, sendo o diagnóstico prénatal estabelecido em 3 (37,5\%) delas. Ventilação mecânica prévia foi necessária em metade dos pacientes e $3(37,5 \%)$ apresentavam choque cardiogênico. Metade dos pacientes estava com infecção sistêmica ativa em tratamento.

Três $(37,5 \%)$ pacientes apresentavam comunicação interatrial restritiva que necessitou de atriosseptostomia (intraútero em um e pós-natal no restante). Nenhum paciente era portador de síndrome genética. Hérnia diafragmática foi corrigida logo após o nascimento em um dos neonatos.

\section{Técnica cirúrgica}

Primeiro estágio (implante de stent em canal arterial e bandagem das artérias pulmonares): Os aspectos técnicos do procedimento híbrido seguiram as diretrizes disponíveis na literatura ${ }^{9,11,12}$. O procedimento foi realizado na sala de hemodinâmica, com material e pessoal treinado em procedimentos cirúrgicos cardíacos neonatais. O procedimento foi realizado sob anes- 
Hincapie MJ, et al. Experiência Inicial de um Novo Centro no Brasil na Abordagem Híbrida para a Síndrome de Hipoplasia do Coração Esquerdo. Rev Bras Cardiol Invas. 2009;17(3):369-77.

tesia geral e a monitorização, por meio de eletrocardiograma contínuo e pressão arterial invasiva. A via de acesso foi a esternotomia mediana e pericardiotomia longitudinal. Após a abertura do pericárdio, ambos os ramos da artéria pulmonar foram amplamente mobilizados e a bandagem desses ramos foi realizada com tira de politetrafluoretileno (W. L. Gore, Newark, Estados Unidos) de $3 \mathrm{~mm}$ ou $4 \mathrm{~mm}$ de espessura por $2 \mathrm{~mm}$ de largura (Figura 1). O ajuste da bandagem foi guiado pela saturação arterial de oxigênio, a fim de mantê-la por volta de $80 \%$, com fração inspirada de oxigênio a $100 \%$. Observou-se aumento de $10 \mathrm{mmHg}$ a $20 \mathrm{mmHg}$ da pressão arterial sistólica após a bandagem. Após a realização da bandagem, um introdutor valvado $6 \mathrm{~F}$ foi inserido pela face lateral direita do tronco da artéria pulmonar. A escolha do tamanho do stent foi guiada por análise angiográfica do canal arterial em relação a sua extensão e a seu diâmetro. Usualmente, foram utilizados stents com $8 \mathrm{~mm}$ de diâmetro por $17 \mathrm{~mm}$ de comprimento (Express LD, Boston Scientific, Natick, Estados Unidos) pré-montados em balão. Fio-guia de 0,035 polegada foi conduzido até a aorta descendente abdominal, passando através do canal arterial. O conjunto balão-stent foi avançado por sobre o fio-guia e posicionado para cobrir toda a extensão do tecido ductal desde a artéria pulmonar até a aorta descendente, sendo liberado com insuflação controlada, respeitando a pressão nominal do balão. Ao final do procedimento, foram realizados controles angiográficos da posição do stent e da bandagem dos ramos pulmonares (Figura 2). Fios de marca-passo epicárdico e dreno mediastinal foram inseridos e o fechamento do tórax foi realizado em todos os pacientes, à exceção de dois, que tiveram instabilidade hemodinâmica e necessidade de drogas inotrópicas em altas doses.
Segundo estágio (reconstrução da aorta e restabelecimento do fluxo pulmonar): No segundo estágio, são realizadas reconstrução da saída do ventrículo esquerdo, ampliação do arco aórtico, retirada do stent do canal arterial e retirada da bandagem das artérias pulmonares (com ou sem plastia das artérias pulmonares), além de ser restabelecido o fluxo pulmonar, com a realização de anastomose entre a veia cava superior e a artéria pulmonar (operação de Glenn bidirecional) ou de anastomose sistêmico-pulmonar (Blalock-Taussig modificado). Por meio de reesternotomia mediana, somente as estruturas necessárias para a realização da operação foram cuidadosamente liberadas de aderências relacionadas à operação anterior. A circulação extracorpórea foi estabelecida por meio da canulação direta da aorta ou do tronco braquicefálico e do átrio direito. Durante o resfriamento, foi realizada a desconexão das artérias pulmonares direitas e esquerdas, ampliando a região referente ao tronco da artéria pulmonar e ao canal arterial. Em hipotermia profunda $\left(20^{\circ} \mathrm{C}\right)$ e parada circulatória total, procedeu-se à retirada do stent do canal arterial, seguida da reconstrução do arco aórtico e aorta distal ao canal com homoenxerto criopreservado ou pericárdio autólogo, evitando grandes áreas de descolamento e tração excessiva. Finalmente, a atriosseptectomia foi realizada ainda em parada circulatória total, e o fluxo pulmonar foi restabelecido durante o reaquecimento. Este último foi construído, preferencialmente, por meio de conexão cavopulmonar bidirecional.

\section{Cuidados pós-operatórios da fase I}

Após a fase I (procedimento híbrido), o paciente foi encaminhado à unidade de pós-operatório entubado e em ventilação controlada. $\mathrm{O}$ objetivo do tratamento

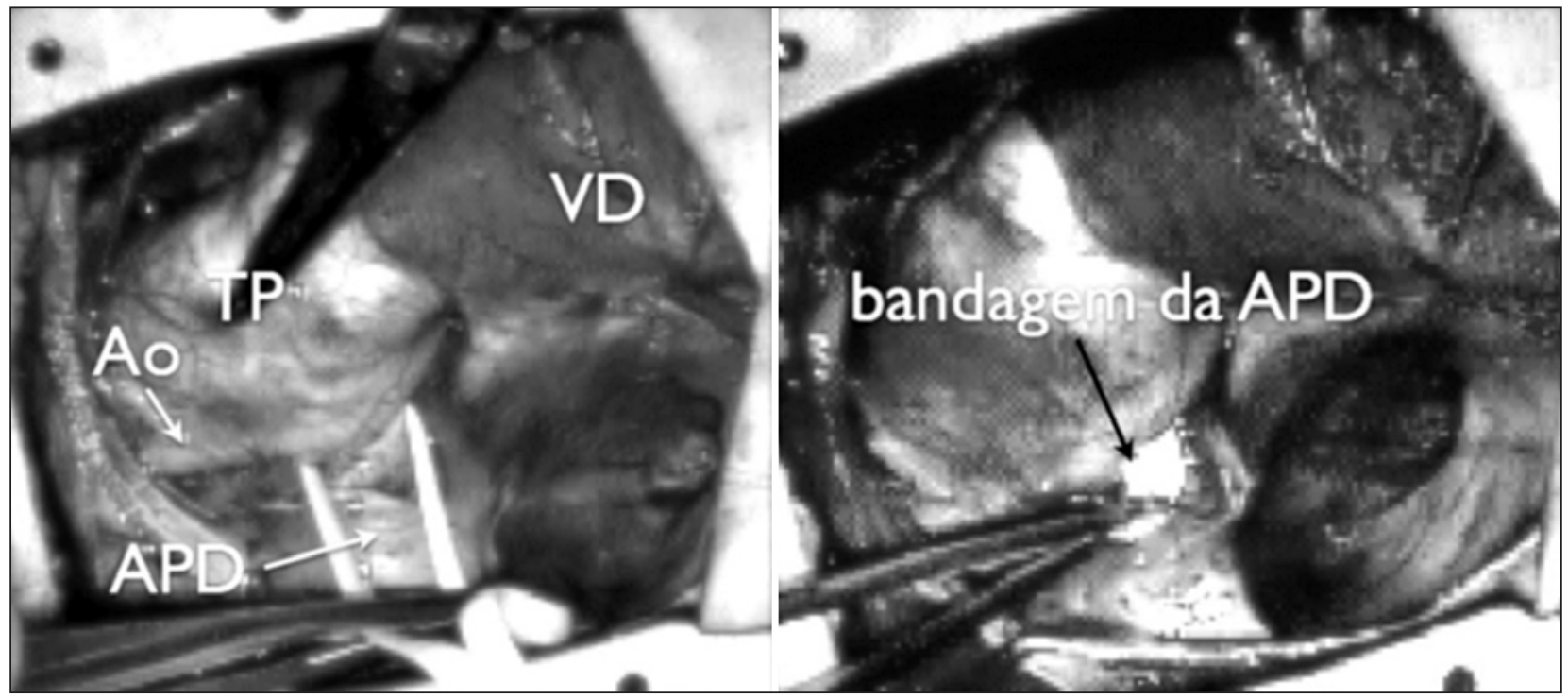

Figura 1 - Aspecto cirúrgico da bandagem da artéria pulmonar direita no procedimento híbrido no tratamento da síndrome de hipoplasia do coração esquerdo. $\mathrm{APD}=$ artéria pulmonar direita; $\mathrm{Ao}=$ aorta; $\mathrm{TP}=$ tronco pulmonar; $\mathrm{VD}=$ ventrículo direito. 


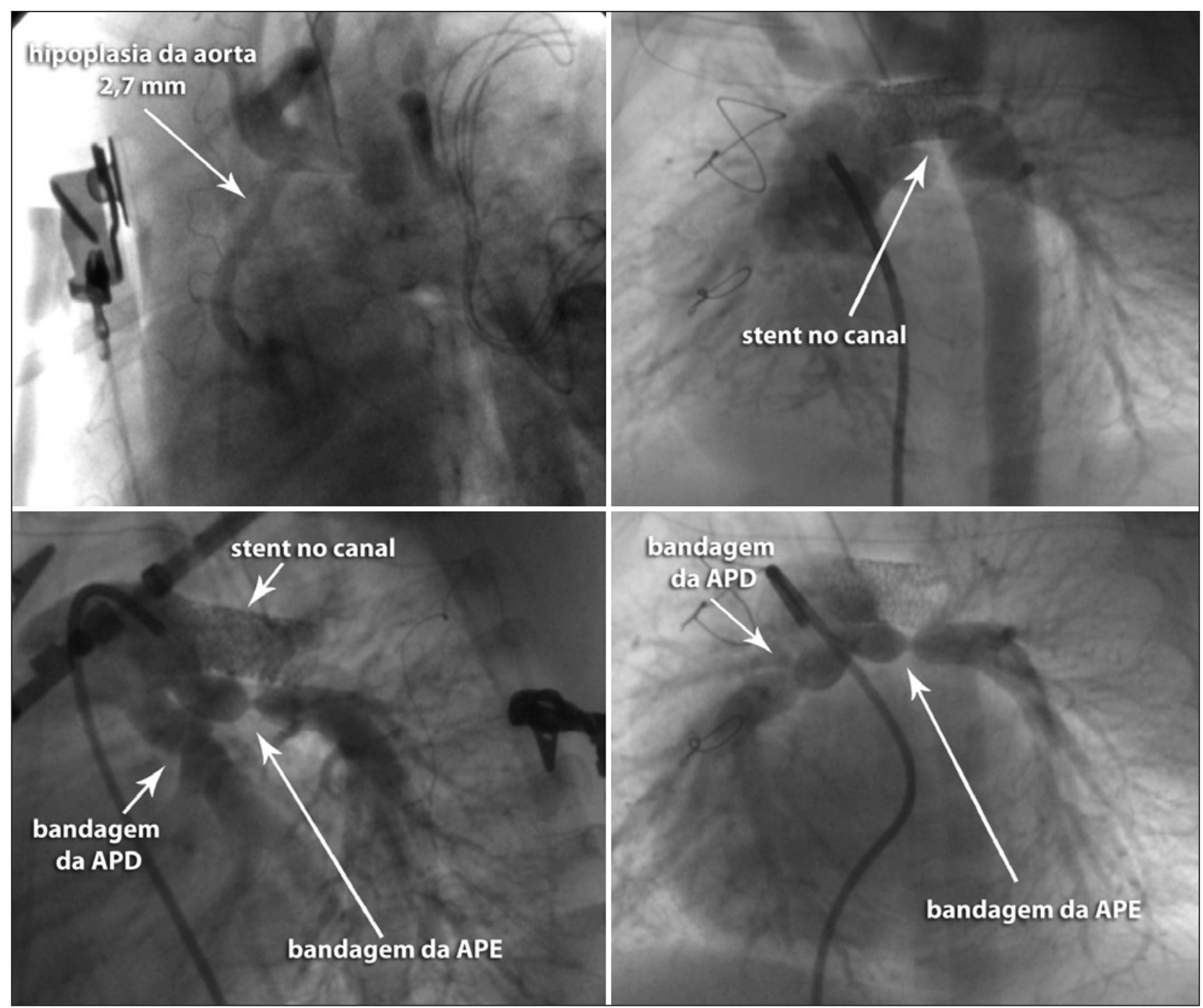

Figura 2 - Angiografia demonstrando aspectos comuns do primeiro estágio do procedimento híbrido na síndrome de hipoplasia do coração esquerdo. $\mathrm{APD}=$ artéria pulmonar direita; $\mathrm{APE}=$ artéria pulmonar esquerda.

no pós-operatório é manter a relação entre fluxos sistêmico e pulmonar próxima a 1, à semelhança do pósoperatório da operação de Norwood convencional. Dessa forma, manipulações da ventilação pulmonar, a fim de elevar a resistência vascular pulmonar, e medicações inotrópicas e vasodilatadoras sistêmicas são realizadas. A realização de ecocardiograma seriado é importante como guia terapêutico, a fim de monitorar função ventricular, grau de eficácia da bandagem das artérias pulmonares e eventuais complicações mecânicas que possam ocorrer. Reintervenções são eventualmente indicadas durante o período pós-operatório em pacientes que evoluam com hiperfluxo pulmonar apesar de manipulações das resistências sistêmica e pulmonar.

\section{RESULTADOS}

As características e a evolução clínica dos pacientes submetidos a procedimento híbrido estão demons- tradas na Tabela 1. A mortalidade hospitalar foi de $37,5 \%$ ( $n=3$ ), sendo um óbito intraoperatório e decorrente de isquemia miocárdica relacionada a comprometimento do fluxo retrógrado para o arco aórtico pela presença do stent no canal arterial. Os outros dois óbitos foram relacionados a septicemia no $72^{\circ}$ dia de pós-operatório em um e a choque cardiogênico prolongado no sexto dia no outro.

Reintervenções foram necessárias em 50\% dos casos $(n=3)$, em dois deles mais de uma vez. A reintervenção mais comum antes da realização do segundo estágio foi a atriosseptostomia por balão com implante de stent na comunicação interatrial (Figura 3). O tempo médio de ventilação após o primeiro estágio foi de $585 \pm 603,54$ horas (0 a 1.200 horas), ou seja, média de 24 dias, e o tempo médio de internação na unidade de terapia intensiva foi de 32 dias (3 a 50 dias). As principais complicações pós-operatórias desses 
TABELA 1

Experiência clínica, procedimentos interestágios e resultados

\begin{tabular}{|c|c|c|c|c|c|}
\hline Paciente & Diagnóstico & $\begin{array}{l}\text { Idade no } \\
\text { procedimento } \\
\text { híbrido (dias) }\end{array}$ & Peso (g) & Reintervenção & Resultado \\
\hline 1 & $\begin{array}{l}\text { VU, DVEVE, TGA, AHT, } \\
\text { infecção, choque }\end{array}$ & 62 & 4.600 & $\begin{array}{l}2{ }^{\circ} \text { estágio: a. Norwood + BT } \\
\text { b. "Takedown" do Glenn + BT }\end{array}$ & Morte súbita \\
\hline 2 & $\begin{array}{c}\text { SHCE, } \\
\text { diagnóstico fetal, HDC }\end{array}$ & 12 & 3.045 & Nenhum & $\begin{array}{r}\text { Óbito durante o } 1^{\circ} \text { estágio, } \\
\text { laboratório de hemodinâmica }\end{array}$ \\
\hline 3 & $\begin{array}{c}\text { SHCE, } \\
\text { diagnóstico fetal }\end{array}$ & 7 & 3.230 & Nenhum & Sépsis, óbito, $72^{\circ} \mathrm{PO}$ \\
\hline 4 & SHCE, infecção, choque & 14 & 3.230 & $\begin{array}{c}\text { Colocação de outro } \\
\text { stent no canal }\end{array}$ & Choque, óbito, $6^{\circ} \mathrm{PO}$ \\
\hline 5 & $\begin{array}{c}\text { SHCE, infecção, } \\
\text { choque, AHT }\end{array}$ & 37 & 3.300 & $\begin{array}{l}\text { a. Atriosseptostomia com balão } \\
\text { b. } 2^{\underline{o}} \text { estágio: Norwood + BT }\end{array}$ & $\begin{array}{l}\text { Hipoxemia, tentativa de } \\
\text { implante de stent na } \\
\text { APE, óbito, laboratório } \\
\text { de hemodinâmica }\end{array}$ \\
\hline 6 & $\begin{array}{l}\text { SHCE, diagnóstico fetal, } \\
\text { IT importante }\end{array}$ & 4 & 2.315 & $\begin{array}{l}\text { a. Atriosseptostomia com balão } \\
\text { b. Colocação de stent na } \\
\text { comunicação interatrial }\end{array}$ & $\begin{array}{c}\text { Internado, esperando } \\
\text { o } 2^{\underline{o}} \text { estágio }\end{array}$ \\
\hline 7 & SHCE, infecção & 12 & 3.935 & Nenhum & $\begin{array}{c}\text { Em casa, esperando } \\
\text { o } 2^{\circ} \text { etágio }\end{array}$ \\
\hline 8 & $\mathrm{SHCE}, \mathrm{AF}, \mathrm{HI}$ & 4 & 3.390 & $\begin{array}{c}\text { Colocação de stent } \\
\text { na comunicação interatrial* }\end{array}$ & $\begin{array}{c}\text { Internado, esperando } \\
\text { o } 2^{\circ} \text { estágio }\end{array}$ \\
\hline
\end{tabular}

* Atriosseptostomia fetal prévia, 32aㅗ semana gestacional. AF = atriosseptostomia fetal; AHT = apresentação hospitalar tardia; APE = artéria pulmonar esquerda; BT = Blalock-Taussig; DVEVE = dupla via de entrada do ventrículo esquerdo; HDC = hérnia diafragmática congênita; $\mathrm{HI}$ = hemorragia intracraniana; IT = insuficiência tricúspide; PO = pós-operatório; SHCE = síndrome de hipoplasia do coração esquerdo; TGA = transposição das grandes artérias; $\mathrm{VU}=$ ventrículo único.

pacientes foram infecção respiratória em $70 \%$, entubação prolongada (superior a sete dias) em 100\%, baixo débito cardíaco em 90\%, e infecção sistêmica em 50\%.

Três crianças aguardam o segundo estágio do procedimento. Duas dessas crianças estão internadas, uma por insuficiência respiratória grave e infecção sistêmica desde a realização do primeiro estágio do procedimento híbrido e outra, desde o período pós-atriosseptostomia com implante de stent, evoluindo com infecção pulmonar. A primeira nunca recebeu alta da unidade de terapia intensiva e a segunda retornou dois meses após a alta do primeiro procedimento.

O segundo estágio foi realizado em dois pacientes, aos 5 e aos 6 meses de idade, respectivamente. O primeiro paciente era portador de ventrículo único funcional, dupla via de entrada do ventrículo esquerdo, discordância ventriculoarterial, ventrículo direito à esquerda, coartação de aorta e comunicação interventricular restritiva. Realizou-se conexão do tronco pulmonar com a aorta ascendente à direita, utilizando remendo de homoenxerto pulmonar, que também serviu para ampliação do arco aórtico e aorta descendente proximal. O stent foi retirado sem dificuldades. O restabelecimento do fluxo pulmonar foi inicialmente cons- truído por conexão cavopulmonar bidirecional. Entretanto, o paciente evoluiu com síndrome de veia cava superior e hipoxemia grave no pós-operatório. Investigação diagnóstica revelou que havia trombose da veia cava inferior, não diagnosticada previamente ao segundo estágio. No quinto dia de pós-operatório, foi submetido a retirada da conexão cavopulmonar e realização de anastomose sistêmico-pulmonar modificada. Evoluiu subsequentemente com septicemia relacionada a pneumonia (entubação prolongada), obtendo alta da unidade de terapia intensiva no $59^{\circ}$ dia. Por ocasião da alta hospitalar ( $\left.72^{\circ} \mathrm{dia}\right)$, apresentou parada cardiorrespiratória súbita, sem fenômenos desencadeantes evidentes, e não respondeu a manobras de reanimação. A necropsia não revelou a causa do óbito, mas a anastomose sistêmico-pulmonar encontrava-se pérvia.

O segundo paciente apresentava a aorta ascendente muito fina, tendo sido seccionada e reimplantada na neoaorta por meio de anastomose terminolateral. As bandagens foram retiradas satisfatoriamente, permitindo a passagem de dilatador de $4 \mathrm{~mm}$ em ambos os ramos. O stent foi retirado sem problemas por meio de secção do tronco pulmonar e endarterectomia de fragmentos distais locados próximos à aorta descendente. 


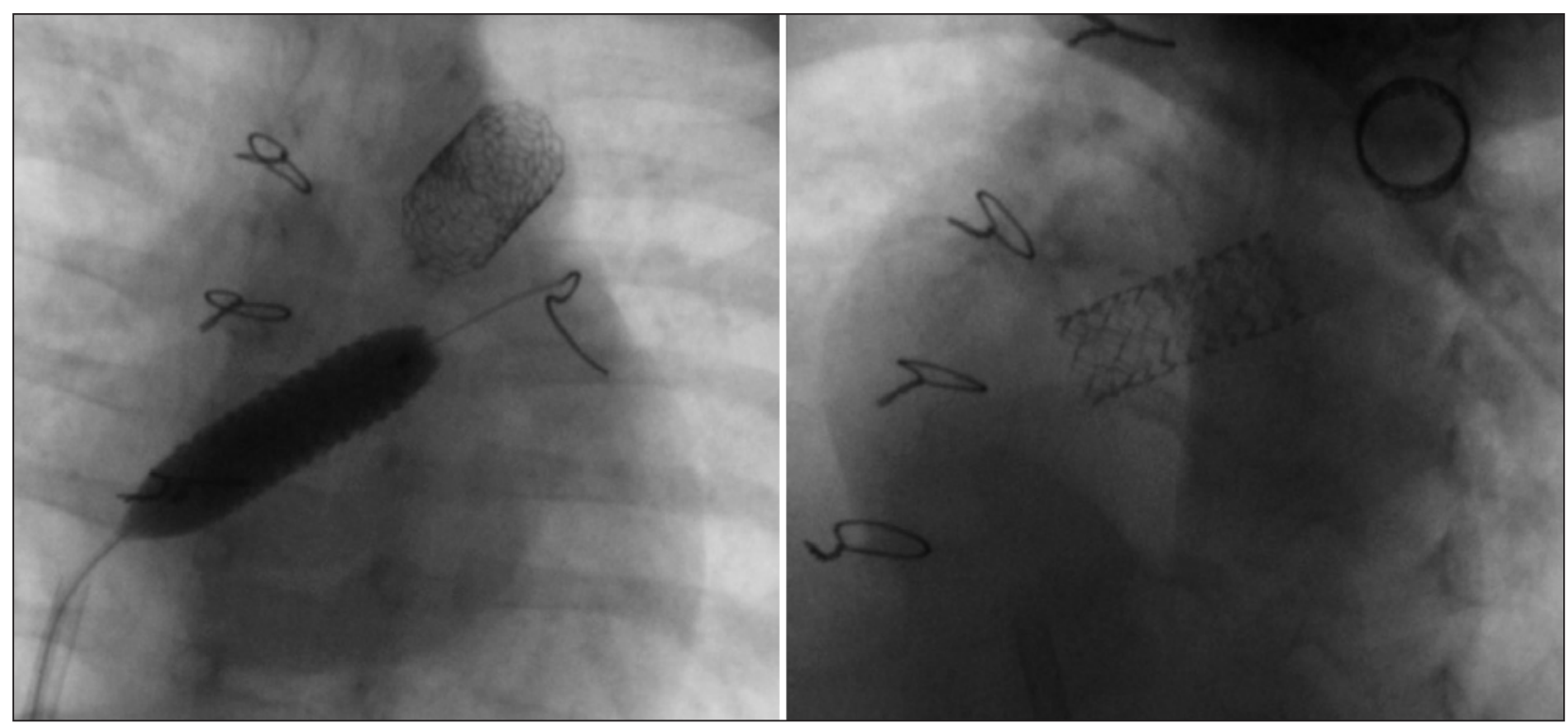

Figura 3 - Angiografia do paciente 6 com comunicação interatrial restritiva submetido a dilatação com balão, seguida de implante de stent.

O fluxo pulmonar foi restabelecido por meio de anastomose sistêmico-pulmonar com tubo de politetrafluoretileno de $4 \mathrm{~mm}$. Evolui hemodinamicamente estável, porém com saturação de oxigênio inferior a 75\%. Como o quadro de hipoxia mantinha-se constante nas primeiras 20 horas de pós-operatório, foi realizado novo ecocardiograma, que sugeriu estenose da artéria pulmonar esquerda, sendo encaminhado o paciente ao laboratório de hemodinâmica para estudo e provável intervenção terapêutica. A estenose da artéria pulmonar esquerda foi confirmada pelo cateterismo cardíaco (Figura 4). Durante o procedimento, houve piora do quadro de choque e hipoxia; o paciente apresentou parada cardíaca, não respondeu às manobras de ressuscitação e faleceu.

\section{DISCUSSÃO}

O procedimento híbrido tem vantagens relacionadas ao fato de ser tecnicamente mais simples e de evitar a circulação extracorpórea neonatal, minimizando as possíveis lesões encefálicas causadas por sucessivos procedimentos com circulação extracorpórea ${ }^{13,14}$. Além disso, permite que a reconstrução do arco aórtico e o estabelecimento da conexão cavopulmonar bidirecional sejam realizados em procedimento único com estruturas mais desenvolvidas. Por fim, permite o crescimento pós-natal de uma porcentagem baixa de ventrículos esquerdos hipoplásicos que possam evoluir para correção biventricular alguns meses após o nascimento.

Entretanto, nessa experiência inicial, essas vantagens não se traduziram em melhores resultados. Apesar de o procedimento ter sido menos complexo inicialmente, o manejo pós-operatório do primeiro está- gio foi difícil, pois o ajuste das bandagens é dinâmico e depende de vários fatores, como tamanho das artérias pulmonares, grau de hipertensão pulmonar inicial e tamanho da comunicação interatrial, entre outros. O ajuste da bandagem é por vezes tão difícil que o uso de bandagem ajustável foi proposto para facilitar o manuseio do balanço entre os fluxos pulmonar e sistêmico necessário nesses $\operatorname{casos}^{15}$. Mesmo sem a utilização da circulação extracorpórea, alguns pacientes evoluíram com reação inflamatória sistêmica, manifestada por temperatura central acima de $38,5^{\circ} \mathrm{C}$, saturação venosa de oxigênio elevada e resistência sistêmica baixa. A estratégia de fechamento tardio do esterno em pacientes instáveis foi necessária em alguns casos. Distúrbios de coagulação são infrequentes e no pósoperatório tardio é importante manter o paciente com antiagregante plaquetário.

A necessidade de reintervenções interprocedimentos não foi insignificante e foi necessária em metade dos pacientes, como observado na maioria das experiências publicadas ${ }^{11,16}$. Número considerável de reintervenções é necessário para otimizar a fisiologia paliativa imposta pela técnica, em especial nos casos de comunicação interatrial restritiva, que necessitam dilatações sucessivas com balão, implante de stent, e uso de balões com lâminas cortantes, em alguns $\operatorname{casos}^{17}$. Além da necessidade de reintervenções na comunicação interatrial, é relatada a necessidade de reintervenções no canal arterial. O gradiente de pressão no canal arterial está comumente relacionado com a cobertura incompleta do canal pelo stent, como ocorreu nas experiências iniciais ${ }^{17}$, mas com o uso de stents mais longos isso foi completamente eliminado. O aparecimento de gradiente de pressão no canal arterial com stent pode ocorrer com a obstrução progressiva pelo 


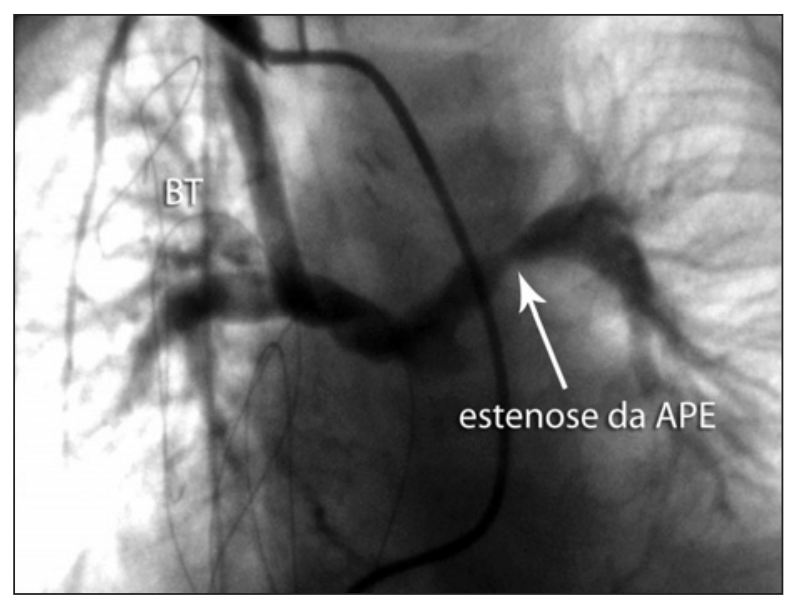

Figura 4 - Angiografia demonstrando estenose da artéria pulmonar esquerda após o segundo estágio (primeiro dia de pós-operatório). $\mathrm{APE}=$ artéria pulmonar esquerda; $\mathrm{BT}=$ Blalock-Taussig.

acúmulo de tecido neointimal, particularmente na presença de canal arterial com anatomia não usual. Em nossa experiência, observamos apenas um caso com estenose precoce na extremidade pulmonar do canal, causada por inadequada cobertura do canal pelo stent. Nesse caso foi necessária a colocação de um segundo stent, com bom resultado tanto anatômico como funcional.

O segundo estágio é uma operação bastante desafiadora para o cirurgião cardiovascular. Trata-se de uma reoperação, com um coração já manipulado previamente, com aderências e presença do stent incorporado à parede do canal, parte do tronco pulmonar e aorta descendente. Em decorrência das dificuldades técnicas, alternativas cirúrgicas foram propostas para minimizar o tempo operatório, como a manutenção do stent e a reconstrução direta do arco aórtico ${ }^{18}$. Em nossos dois casos submetidos ao segundo estágio, não tivemos problemas com os stents, os quais foram facilmente retirados. Quando a reconstrução da neoaorta e do arco transverso necessita de tecido adicional, temos utilizado homoenxerto criopreservado para esse fim. O fato de os tecidos estarem mais fixos, pela reação inflamatória e pelas aderências causadas pela manipulação na primeira cirurgia, não permite grandes mobilizações dessas estruturas, o que dificulta bastante realizar o segundo estágio desse procedimento sem a utilização de tecido autólogo adicional.

A utilização de politetrafluoretileno para realização de bandagem das artérias pulmonares é preconizada por alguns autores ${ }^{17}$, pois acredita-se que esse material cause pouca reação cicatricial local e que, após a retirada da bandagem, as artérias ganhem tamanho adequado e proporcional ao segmento distal. Pilla et al. ${ }^{11}$ relataram a necessidade de dilatação e implante de stent em todos os quatro casos submetidos ao segundo estágio. Chama a atenção que os referidos autores ${ }^{11}$ utilizaram pericárdio bovino ou tira de silicone para a realização da bandagem. Já Pizarro et al. ${ }^{17}$, que utilizam politetrafluoretileno para a realização da bandagem, relataram pequena incidência de estenose das artérias pulmonares, com apenas dois casos em que foi necessária a plastia da artéria pulmonar esquerda intraoperatória. Em nossos pacientes, observamos discreta reação inflamatória no local da bandagem, sendo muito fácil a retirada da bandagem das artérias pulmonares. Após a retirada da banda de politetrafluoretileno, a artéria adquiriu diâmetro semeIhante ao do segmento distal, não necessitando ampliação da artéria. No segundo paciente submetido ao segundo estágio observamos desenvolvimento pequeno da artéria pulmonar esquerda. Embora tenha sido julgada não necessária a sua ampliação no período intraoperatório, o paciente evolui com hipoxemia persistente no período pós-operatório imediato e a angiografia pós-operatória demonstrou estenose no local da bandagem. A necessidade de plastia dos ramos pulmonares, distorcidos pela presença da bandagem, pode aumentar a complexidade cirúrgica no segundo estágio, elevando o risco intraoperatório. $\mathrm{Na}$ experiência de Pizarro et al. ${ }^{17}$, dentre os dois óbitos relatados, a causa de um deles foi decorrente de hemorragia grave relacionada a plastia intraoperatória da artéria pulmonar esquerda.

No segundo estágio, a realização da anastomose cavopulmonar facilita o manuseio pós-operatório dos pacientes com fisiologia univentricular. O ideal é que o segundo estágio seja feito após o quinto mês de vida, quando a vascularização pulmonar está bem desenvolvida e a veia cava apresenta tamanho adequado, embora a operação do Glenn em idades inferiores possa ser realizada com bastante segurança ${ }^{19,20}$. $\mathrm{Na}$ presença de comunicação interatrial restritiva, observamos grande dificuldade no manuseio clínico dessas crianças até a realização do segundo estágio no tempo recomendado. Mesmo com a realização de sucessivas atriosseptostomias por balão com ou sem implante de stent, a grande dificuldade em conseguir uma comunicação interatrial efetiva, a fim de obter oxigenação adequada, é bastante evidente. Pizarro et al. ${ }^{17}$ referem a necessidade de desfazer um de seus casos, com realização de Blalock-Taussig modificado por hipoxia persistente. Em nossa experiência clínica, os casos que se apresentaram com comunicação interatrial restritiva necessitaram sucessivas atriosseptostomias, inclusive com implante de stent, para que pudessem atingir a idade ideal preconizada para o segundo estágio. Nosso primeiro caso, embora tenha sido submetido ao segundo estágio aos 6 meses de idade, não tolerou a anastomose cavopulmonar realizada. A retirada da operação de Glenn e a realização de Blalock-Taussig modificado com tubo de Gore-Tex ${ }^{\mathrm{TM}}$ (W. L. Gore, Newark, Estados Unidos) $4 \mathrm{~mm}$ decorreram da síndrome de cava superior, pois nesse caso havia obstrução da cava inferior em consequência do 
cateter venoso, levando a uma fisiologia semelhante à cavopulmonar total quando da realização da anastomose da cava superior com a artéria pulmonar direita. Os quatro casos iniciais indicados para procedimento híbrido foram escolhidos como primeira opção de tratamento para a síndrome de hipoplasia do coração esquerdo e os demais, por serem considerados casos de alto risco. Entre as condições consideradas de alto risco, como prematuridade, baixo peso ao nascer, presença de outras anormalidades não-cardíacas e síndromes genéticas associadas, considerou-se, em nossa experiência, infecção ativa em evolução, apresentação tardia para o tratamento cirúrgico e presença de insuficiência da valva tricúspide grave como indicação do procedimento híbrido.

Com a experiência clínica acumulada, os resultados do primeiro tempo do procedimento apresentaram significativa melhora. Os óbitos em nossa experiência ocorreram no segundo, terceiro e quarto pacientes. Isso reforça o impacto da curva de aprendizado desse procedimento, tanto no manuseio intraoperatório no laboratório de hemodinâmica como nos cuidados pós-operatórios na unidade de terapia intensiva pediátrica.

Nosso estudo apresenta algumas limitações. Trata-se de um estudo retrospectivo, com pequeno número de pacientes e de uma experiência inicial, cuja importância da curva de aprendizado é conhecida. Isso não nos permite fazer qualquer comparação entre esse procedimento e a operação de Norwood convencional. O resultado não-satisfatório aqui apresentado pode estar relacionado muito mais à dificuldade de manuseio do neonato como um todo, pelas dificuldades de treinamento da equipe, condições de trabalho e estado pré-operatório do paciente, que propriamente decorrente do procedimento. Para se obter o resultado ideal no tratamento do ventrículo único funcional é necessário maior investimento nesses pontos deficientes, além da incorporação de novas tecnologias.

\section{CONCLUSÃO}

A operação de Norwood convencional ainda é considerada a primeira opção de tratamento para a síndrome de hipoplasia do coração esquerdo pela maioria dos serviços de cardiologia pediátrica ${ }^{3}$. A indicação do procedimento híbrido tem papel importante nos casos considerados de "alto risco"16 e talvez possa ser a primeira escolha em grupos dedicados exclusivamente a esse tipo de procedimento, uma vez que esses grupos são os que apresentam melhor resultado na literatura ${ }^{10}$. Os grupos que sempre consideraram preferencialmente o procedimento híbrido como primeira opção de tratamento para a síndrome de hipoplasia do coração esquerdo podem ter seus resultados explicados pela convicção no método, pela dedicação e pela experiência com essa técnica. Até o presente momento, não temos nenhuma evidência clínica da superioridade de um procedimento sobre o outro no tratamento da síndrome de hipoplasia do coração esquerdo, ficando a escolha baseada na preferência e no resultado individual de cada equipe.

De qualquer forma, a escolha pelo procedimento híbrido não deve ser justificada por se tratar de um procedimento mais simples, menos invasivo ou pelo resultado ruim obtido com a operação de Norwood convencional pelo serviço.

\section{CONFLITO DE INTERESSES}

Os autores declararam inexistência de conflito de interesses.

\section{REFERÊNCIAS BIBLIOGRÁFICAS}

1. Jenkins PC, Flanagan MF, Sargent JD, Canter CE, Chinnock $\mathrm{RE}$, Jenkins KJ, et al. A comparison of treatment strategies for hypoplastic left heart syndrome using decision analysis. J Am Coll Cardiol. 2001;38(4):1181-7.

2. Atik FA. Applied physiology to the contemporary management of the neonate with hypoplastic left heart syndrome. Arq Bras Cardiol. 2006;87(3):e16-26.

3. Wernovsky G, Ghanayem N, Ohye RG, Bacha EA, Jacobs JP, Gaynor JW, et al. Hypoplastic left heart syndrome: consensus and controversies in 2007. Cardiol Young. 2007;17 Suppl 2: 75-86.

4. Jacobs JP, Jacobs ML, Maruszewski B, Lacour-Gayet FG, Clarke DR, Tchervenkov $\mathrm{Cl}$, et al. Current status of the European Association for Cardio-Thoracic Surgery and the Society of Thoracic Surgeons Congenital Heart Surgery Database. Ann Thorac Surg. 2005;80(6):2278-83.

5. Gaynor JW, Mahle WT, Cohen MI, Ittenbach RF, DeCampli WM, Steven JM, et al. Risk factors for mortality after the Norwood procedure. Eur J Cardiothorac Surg. 2002;22(1): 82-9.

6. Rasiah SV, Ewer AK, Miller P, Wright JG, Barron DJ, Brawn WJ, et al. Antenatal perspective of hypoplastic left heart syndrome: 5 years on. Arch Dis Child Fetal Neonatal Ed. 2008;93(3):F192-7.

7. Silva JP, Fonseca L, Baumgratz JF, Castro RM, Franchi SM, Lianza AC, et al. Hypoplastic left heart syndrome: the report of a surgical strategy and comparative results of Norwood $\mathrm{x}$ Norwood-Sano approach. Rev Bras Cir Cardiovasc. 2007; 22(2):160-8.

8. DATASUS. Procedimentos hospitalares do SUS - por local de internação - Brasil. Brasília: Ministério da Saúde; 2007.

9. Gibbs JL, Wren C, Watterson KG, Hunter S, Hamilton JR. Stenting of the arterial duct combined with banding of the pulmonary arteries and atrial septectomy or septostomy: a new approach to palliation for the hypoplastic left heart syndrome. Br Heart J. 1993;69(6):551-5.

10. Galantowicz M, Cheatham JP, Phillips A, Cua CL, Hoffman TM, Hill SL, et al. Hybrid approach for hypoplastic left heart syndrome: intermediate results after the learning curve. Ann Thorac Surg. 2008;85(6):2063-70.

11. Pilla CB, Pedra CA, Nogueira AJ, Jatene M, Souza LC, Pedra $S R$, et al. Hybrid management for hypoplastic left heart syndrome: an experience from Brazil. Pediatr Cardiol. 2008; 29(3):498-506.

12. Galantowicz M, Cheatham JP. Lessons learned from the development of a new hybrid strategy for the management 
Hincapie MJ, et al. Experiência Inicial de um Novo Centro no Brasil na Abordagem Híbrida para a Síndrome de Hipoplasia do Coração Esquerdo. Rev Bras Cardiol Invas. 2009;17(3):369-77.

of hypoplastic left heart syndrome. Pediatr Cardiol. 2005; 26(3):190-9.

13. Bacha EA, Daves S, Hardin J, Abdulla RI, Anderson J, Kahana $M$, et al. Single-ventricle palliation for high-risk neonates: the emergence of an alternative hybrid stage I strategy. J Thorac Cardiovasc Surg. 2006;131(1):163-71.

14. Alsoufi B, Bennetts J, Verma S, Caldarone CA. New developments in the treatment of hypoplastic left heart syndrome. Pediatrics. 2007;119(1):109-17.

15. Assad RS, Zamith MM, Silva MF, Thomaz PG, Miana LA, Guerra VC, et al. A novel adjustable pulmonary artery banding system for hypoplastic left heart syndrome. Ann Thorac Surg. 2007;84(6):2081-4.

16. Pizarro C, Derby CD, Baffa JM, Murdison KA, Radtke WA. Improving the outcome of high-risk neonates with hypoplastic left heart syndrome: hybrid procedure or conventional surgical palliation? Eur J Cardiothorac Surg. 2008;33(4): 613-8.
17. Pizarro C, Murdison KA, Derby CD, Radtke W. Stage II reconstruction after hybrid palliation for high-risk patients with a single ventricle. Ann Thorac Surg. 2008;85(4):1382-8.

18. Caldarone CA, Honjo O, Benson LN, Van Arsdell GS. Modification of stage II procedure after hybrid palliation (bilateral pulmonary artery banding and ductal stenting) for hypoplastic left-sided heart syndrome: Modified arch reconstruction with retained stented ductus patch. J Thorac Cardiovasc Surg. 2007;134(6):1588-9.

19. Jaquiss RD, Siehr SL, Ghanayem NS, Hoffman GM, Fedderly RT, Cava JR, et al. Early cavopulmonary anastomosis after Norwood procedure results in excellent Fontan outcome. Ann Thorac Surg. 2006;82(4):1260-5.

20. Jaquiss RD, Ghanayem NS, Hoffman GM, Fedderly RT, Cava $J R$, Mussatto KA, et al. Early cavopulmonary anastomosis in very young infants after the Norwood procedure: impact on oxygenation, resource utilization, and mortality. J Thorac Cardiovasc Surg. 2004;127(4):982-9. 